\title{
Philosophiques
}

\section{L'importance du mécanisme de projection imaginatif au sein de la démarche éthique spinozienne}

\section{Andrea Zaninetti}

Volume 29, numéro 1, printemps 2002

Spinoza sous le prisme de son anthropologie

URI : https://id.erudit.org/iderudit/009566ar

DOI : https://doi.org/10.7202/009566ar

Aller au sommaire du numéro

Éditeur(s)

Société de philosophie du Québec

ISSN

0316-2923 (imprimé)

1492-1391 (numérique)

Découvrir la revue

Citer cet article

Zaninetti, A. (2002). L'importance du mécanisme de projection imaginatif au sein de la démarche éthique spinozienne. Philosophiques, 29(1), 99-105.

https://doi.org/10.7202/009566ar d'utilisation que vous pouvez consulter en ligne.

https://apropos.erudit.org/fr/usagers/politique-dutilisation/ 


\title{
L'importance du mécanisme de projection imaginatif au sein de la démarche éthique spinozienne
}

\author{
ANDREA ZANINETTI \\ Université de Montréal \\ azaninetti@hotmail.com
}

\begin{abstract}
RÉSUMÉ. - Contrairement à Descartes, Spinoza regroupe sous le terme « imagination », des processus cognitifs que l'on s'attendrait ordinairement à voir distingués, soit la connaissance sensible et l'activité fantasmatique. Quelle est la raison profonde de leur unification ? Dans cet article, nous tenterons de répondre à cette question, en montrant tout d'abord que ces processus cognitifs partagent une caractéristique commune, soit leur dynamique projective (que nous nommons «mécanisme de projection imaginatif»). Après avoir défini les propriétés principales et les limites de ce mécanisme et avoir montré la manière dont celui-ci s'exprime, nous nous demanderons pourquoi il est dans l'intérêt de tout homme de le dépasser. Ce dernier point, fera ressortir la place centrale que ce mécanisme occupe au sein de la démarche éthique spinozienne.
\end{abstract}

\begin{abstract}
In contrast to Descartes, Spinoza amalgamates under the term 'imagination' cognitive processes that one would normally expect to be contradistinguished, i.e., sensitive knowledge and the activity of the fantasy. What is the underlying reason for this unification ? In this paper, I intend to answer this question by first showing that these cognitive processes share a common feature, that of their projective dynamics - which is referred to here as the "imaginative projection mechanism". After showing the main properties and the limits of this mechanism, as well as the way it realizes itself, I will ask why it is in the interest of any human being to transcend it. This last point will highlight the central place occupied by this mechanism within the Spinozistic ethical process.
\end{abstract}

"Ceux donc qui croient parler, se taire ou faire quoi que ce soit en vertu d'un libre décret de l'esprit, rêvent les yeux ouverts " ${ }^{1}$. Ainsi s'achève le long scolie de la proposition II du de Affectibus ; sous l'apparente légèreté de cette déclaration, le lecteur averti décèlera peut-être une pointe acérée, dirigée contre la conception cartésienne du libre-arbitre. Il est fort probable cependant que Spinoza n'ait point voulu viser intentionnellement Descartes dans cet extrait, ce qui n'ôte rien au demeurant à son caractère profondément anti-cartésien. Car Spinoza nous dit ici quelque chose que Descartes a toujours repoussé avec vigueur : l'expérience de la liberté est un rêve éveillé, autrement dit, une illusion. Le terme "rêve " suggère d'emblée qu'une parenté étroite existe entre cette expérience et le songe nocturne. Comme l'illusion de la liberté est indis-

1. Toutes les citations sont tirées de: Spinoza, B., Oeuvres complètes, texte nouvellement traduit ou revu, présenté et annoté par Roland Caillois, Madeleine Francès et Robert Misrahi, Paris, Gallimard, 1954, 1578 p. (Bibliothèque de la Pléiade \# 108). 
sociable de la dynamique imaginative, cette parenté concerne tous les processus cognitifs que cette dynamique englobe. Ceci est possible parce qu'il n'existe pas, chez Spinoza, de démarcation épistémologique permettant de différencier un rêve " les yeux fermés ", d'un rêve « les yeux ouvert " ${ }^{2}$. Cela tient à sa conception très particulière de l'imagination, qui met sur le même plan l'activité fantasmatique et la connaissance sensible. Qu'elles soient mises sur le même plan, ne signifie pas pour autant qu'elles soient confondues : Spinoza distingue en effet implicitement entre une activité fantasmatique qui est indirectement conditionnée par l'objet extérieur qu'elle représente, puisqu'elle procède du contact spontané (spontaneo suo motu) des parties fluides du corps sur les traces imprimées antérieurement par celles-ci dans les parties molles (sous l'effet d'un corps extérieur), et la connaissance sensible proprement dite qui elle, est directement déterminée par les corps extérieurs qu'elle représente. Cette distinction se retrouve dans la proposition $17 \mathrm{du}$ de Mente : la première démonstration est consacrée à la connaissance sensible proprement dite et la seconde à l'activité fantasmatique, le corollaire intercalé entre les deux assurant leur transition. Le choix d'introduire ces dernières dans deux démonstrations séparées, indique clairement que Spinoza les considère comme deux mécanismes cognitifs distincts, mais le fait que ceux-ci se retrouvent tous deux au sein de la même proposition, démontre que ces mécanismes doivent être considérés comme étant de nature identique. Spinoza ne nous donne aucun critère permettant de différencier intérieurement ces mécanismes, contrairement à Descartes, qui dans la sixième Méditation les hiérarchise en fonction de la vivacité de leurs représentations (les objets extérieurs sont, pour Descartes, " mieux » perçus par les sens que par l'imagination). La différenciation externe de ces mécanismes, c'est-à-dire la relation immédiate ou médiate que leurs représentations entretiennent avec les corps extérieurs qui les déterminent, n'est pas une raison suffisante pour les considérer comme qualitativement distincts. Au contraire, ce critère extrinsèque, s'il avait été développé plus en détail ou traité dans des propositions séparées, aurait probablement détourné le lecteur de l'Ethique de l'essentiel, soit, que les représentations fantasmatiques et sensibles ont une parenté étroite, du fait que toutes deux, témoignent plutôt du corps de celui qui les forme que des corps extérieurs qui les déterminent.

Ceci le lecteur l'apprend en confrontant la proposition $17 \mathrm{du}$ de Mente à celle qui la précède, soit la proposition 16 : cette dernière permet en effet de comprendre pourquoi l'activité fantasmatique et la connaissance sensible ne sont pas traitées séparément, bien qu'elles soient différenciées théoriquement. Par cette confrontation, le lecteur peut saisir concrètement de quelle manière Spinoza, ainsi qu'il l'annonce dans la préface du de Mente, entend restreindre

2. Non seulement l'ensemble des passions peuvent être considérées comme des rêves éveillés, mais en vertu de la parenté existant entre tous les processus cognitifs de l'imagination, elle peuvent également être identifiées à des "sortes de délires » (E, IV, 44, scolie.), entrainant ainsi une extension du pathologique à ce qui n'est point ordinairement considéré comme tel. 
le champ théorique de l'Ethique à ce qui peut « nous conduire comme par la main à la connaissance de l'Esprit humain et de sa béatitude suprême. » Cette confrontation permet de déceler un subtil compromis entre, d'une part, les exigences de l'analyse scientifique et, d'autre part, celles de la démarche éthique. Alors que la première tend naturellement à séparer les objets du savoir en autant de champs d'investigations qui n'ont d'autre fin qu'eux-mêmes, la seconde, au contraire, cherche au sein de ce dernier les dénominateurs communs qui servent sa propre finalité. Dans l'exemple qui nous occupe présentement, le dénominateur commun existant entre l'activité fantasmatique et la connaissance sensible se trouve dans la proposition 16 et plus particulièrement dans son deuxième corollaire. C'est dans ce dernier que l'on apprend que les « idées que nous avons des corps extérieurs indiquent plutôt la constitution de notre corps que la nature des corps extérieurs. ${ }^{3}$ On ne saurait trop insister sur le " plutôt ", puisque ce simple terme souligne une spécificité importante de l'imagination, soit son caractère projectif, qui constitue précisément le dénominateur commun de tout ce qui est englobé sous le terme "imagination ». On peut même aller jusqu'à conjecturer que la raison de l'unification sous ce terme de mécanismes cognitifs aussi divers que le rêve, la connaissance sensible et l'imagination au sens étroit, réside précisément dans le fait qu'ils partagent tous ce caractère projectif. Cette unification pourrait en ce sens être interprétée comme un avertissement de ne pas se perdre dans la différenciation théorique de ces mécanismes cognitifs, mais au contraire de se concentrer sur le message éthique que leur unification suggère, message qui pourrait se résumer comme suit : les hommes vivent communément dans le monde clos de leurs propres projections.

L'Appendice du de Deo - auquel se réfère Spinoza dans le corollaire précédemment mentionné - fournit des précisions importantes sur la nature du mécanisme de projection imaginatif. Trois caractéristiques essentielles s'en dégagent : premièrement, ce mécanisme est inconscient, l'inconscience de ce dernier découlant de l'ignorance des causes qui le déterminent. Deuxièmement, ce mécanisme est différentiel, en ce sens que les projections qu'il déploie, épousent chaque fois la coloration particulière du tempérament (ingenium) de celui qui imagine, ceci impliquant qu'il y a autant de projections que de tempéraments particuliers. Troisièmement, ce mécanisme est clos, en ce sens que chacune de ses projections se déploie en circuit fermé $e^{4}$ (même si au regard des causes qui déterminent ces dernières, on peut à bon droit le considérer comme un circuit « ouvert»). Ainsi le mécanisme imaginatif peut être défini à la fois comme inconscient, différentiel et clos.

Ces trois termes sont liés mutuellement. L'inconscience du mécanisme imaginatif est indissociable de celle de son caractère différentiel mais également par extension de celle de son cloisonnement. Mais à la différence du

3. Nous soulignons.

4. Autrement dit, sans avoir conscience de l'existence des autres projections. 
caractère différentiel qui, inconscient de lui-même ou non, demeure indépassable (puisque c'est un fait qu'il y a des essences singulières qualitativement distinctes), le caractère clos du mécanisme, lui, peut être dépassé, s’il devient conscient de soi. C'est le deuxième genre de connaissance, soit la connaissance par la Raison, qui rend conscient ce mécanisme, en permettant de considérer simultanément $(\text { simul })^{5}$ plusieurs choses singulières (alors que l'imagination est réduite à la perception d'une diversité éclatée), ce qui rend possible une comparaison entre elles : cette comparaison fait ressortir simultanément le caractère commun des choses comparées et leur caractère différentiel. Or ce qui est digne d'intérêt, c'est que la perception du caractère différentiel des choses, coïncide exactement, selon nous, avec la neutralisation de ce dernier. En effet, c'est au moment où un individu perçoit par la Raison que toute chose a une essence singulière, qu'il prend conscience du même coup de la particularité de sa propre essence et surtout que celle-ci était antérieurement projetée sur le monde extérieur : dès lors, le caractère différentiel de son essence singulière est neutralisé, dans le sens où celle-ci ne déploie plus inconsciemment sur le monde le produit de sa particularité (soit ses projections) ${ }^{6}$ mais au contraire, met sa particularité au service de la formation de notions communes, qui comme leur nom l'indique sont perçues par tous de façon identique.

Nous avons énuméré jusqu'à présent les caractéristiques générales du mécanisme de projection imaginatif et évoqué la possibilité de son dépassement par le deuxième genre de connaissance. Nous nous attarderons maintenant sur la manière dont ce mécanisme s'exprime. Comme ce dernier est neutralisé dès qu'il devient conscient de lui-même, on peut d'emblée poser que c'est l'ignorance qui rend possible son déploiement. Cette ignorance porte sur les causes qui déterminent ce mécanisme. Voyons ceci de plus près en se servant d'un exemple très spinozien. Si le corps de Pierre affecte le corps de Paul, l'idée que Paul aura du corps de Pierre indiquera plutôt l'essence de son propre corps que celle du corps qui l'affecte. Puisque l'idée que Paul aura de son propre corps a été déterminée par une cause extérieure (le corps de Pierre), elle est un effet de cette dernière. Or ceci l'idée imaginative l'ignore. De ce fait elle ignore également le caractère hybride de sa réalité objective, représentant la rencontre des deux corps dans la conscience de celui qui imagine, comme un tout uniforme. Cette double ignorance, entraîne une inver-

5. E, II, 39, scolie.

6. Plus précisément, ces projections continuent à être déployées mais accompagnées des idées de la Raison qui excluent l'existence de ce que les premières représentent. C'est cette rectification de l'imagination par la Raison qui neutralise le caractère projectif de celle-là. Le promeneur imagine le soleil à 200 pieds de lui mais il rectifie spontanément cette perception à l'aide d'idées de la Raison (dans le cas présent, empruntées à l'astronomie), qui lui indiquent la véritable distance du soleil par rapport à la terre. Dans un sens donc, il n'est pas faux de dire que les projections ne se déploient plus une fois rectifiées par la Raison, puisque dans l'exemple du soleil, il est évident sa distance imaginaire n'est dans un sens plus perçue, une fois son existence exclue par les idées rationnelles. 
sion de la perception de l'ordre causal de la Nature. Puisque l'imagination ignore qu'elle est un effet, elle se prend pour une cause. Puisqu'elle ignore le caractère hybride de la réalité objective de ses idées, dont la majeure partie est de nature projective, elle va spontanément croire que ces dernières représentent le monde tel qu'il est vraiment. Ainsi prend forme le rêve éveillé de l'imagination. Rêve de liberté tout d'abord, qui découle de l'illusion d'être une cause première, mais aussi rêve d'un monde qualitativement ordonné qui serait adapté à nos besoins. Ce rêve comme Spinoza le montre dans l'Appendice du de Deo, ne connaît point de limite à son extension : même les dieux sont conçus inconsciemment comme des êtres soumis à la dynamique imaginative, c'est-à-dire comme des répliques plus puissantes de ceux qui les ont imaginés. Le paradoxe de cette dynamique, réside dans le fait que les personnes qui lui sont soumises, sont conditionnées par la Nature à soumettre cette dernière à leur propres besoins. Tout en étant de simples jouets du monde extérieur, elles sont coupées de ce dernier, et s'imaginent au contraire être libres et en contact direct avec la réalité. Seules les personnes qui parviennent à s'intérioriser ${ }^{7}$ ou, ce qui revient au même, à former des idées adéquates, perçoivent le monde tel qu'il est, soit un monde où chaque essence a sa place et où une authentique altérité est possible (même si elle ne peut être pensée sans l'identité divine).

Une question peut légitimement être posée ici, celle de savoir pour quelle raison il est dans notre intérêt de quitter le monde de projections dans lequel, selon Spinoza, nous vivons tous dès la naissance. Sa réponse implique le pourquoi de la démarche éthique spinozienne.

Une des réponses possibles - celle de Deleuze en l'occurrence ${ }^{8}$ — insiste sur le fait que la dynamique imaginative nous soumet inévitablement, à la longue, à de nombreuses mauvaises rencontres, qui finissent par avoir raison du rapport constitutif de notre essence : d'où l'importance de sortir au plus vite de cet état hasardeux qu'est la vie soumise à l'imagination. Cette réponse, qui se concentre sur l'absence de choix caractérisant la vie imaginative et sur les conséquences négatives qui en découlent n'est point convaincante à notre avis, car elle postule le triomphe des mauvaises rencontres sur les bonnes, alors que le contraire pourrait logiquement tout aussi bien être postulé : en effet il n'y a aucune raison, pour que le rapport constitutif de mon essence ne bénéficie pas de bonnes rencontres qui rééquilibrent les dommages causés par les mauvaises et ceci indéfiniment.

Une seconde réponse, que nous défendons, pose au contraire que c'est le caractère projectif de l'imagination qui est éminemment destructeur. Regardons celui-ci de plus près. La dynamique d'une personne soumise à l'imagination consiste à désirer dans le monde extérieur les qualités positives

7. Ibid.

8. Deleuze, Gilles, Spinoza et le problème de l'expression, Paris, Les Éditions de Minuit, 1968, pp. 214-233. 
qu'elle y a projeté. Une projection positive a lieu lorsqu'une partie de la chose extérieure rencontrée - et ce ne peut être qu'une partie - s'accorde avec l'essence du corps de celui qui la rencontre. Une fois cet accord réalisé, la personne s'efforcera de le conserver et s'il est le seul point commun de la rencontre, elle s'efforcera de nier les points de divergence, pour ne se concentrer que sur lui seul. Si cet accord vient à disparaître, elle s'efforcera de le reproduire comme auparavant et détruira tout ce qui l'en empêche. Bien que Spinoza comprenne sous le terme de chose (res), à la fois les choses matérielles, les êtres animés dépourvus de raison et les hommes, il est clair que dans la partie de l'Ethique consacrée à la dynamique imaginative, soit le de Affectibus, ce terme finit par ne désigner que les hommes ${ }^{9}$ (à partir de la démonstration de la proposition 21 pour être précis). Ceci a son importance, car cela signifie que les relations intersubjectives sont au coeur de la démarche éthique spinozienne. Ainsi, la chose en face de moi peut bien entendu être une pierre, elle peut me tomber dessus, me fracasser le crâne et finir par me tuer ; Spinoza inclut la possibilité de telles rencontres, mais il veut que l'on porte plus spécifiquement notre attention sur une chose semblable à nous-mêmes, c'est-à-dire un autre homme, qui va déployer ses stratégies d'appropriation sur notre personne ou résister à celles que l'on déploie sur lui, contrairement à ce que peut faire la pierre. Dans les relations intersubjectives, le mécanisme de projection est inévitablement destructeur, parce que chaque personne s'efforce, autant qu'elle peut, d'aimer dans l'autre la totalité d'elle-même et d'haïr ce qui la nie en partie ou en totalité. Comme l'autre ne convient que partiellement au rapport qui définit notre essence ${ }^{10}$, et qu'il n'entend pas être nié dans les parties qui ne nous conviennent pas chez lui (puisqu'au contraire il va s'efforcer de faire convenir ces parties avec une chose extérieure), cet autre va nécessairement nous haïr pour l'avoir nié et s'efforcera donc de nous détruire, en partie du moins. Ainsi et c'est le point capital, la destruction de notre essence est nécessairement provoquée par nous-mêmes, puisque nous voulons forcer les autres à vivre selon notre propre naturel (ou complexion corporelle) et que ces derniers agissent de même avec nous. Ce qui distingue donc la rencontre d'un homme d'avec celle d'une pierre, c'est que dans le premier cas, les efforts d'appropriation engendrent un ensemble de réponse dans la chose qu'on désire s'approprier, qui multiplie les risques rétroactifs de destruction dans notre propre essence et par conséquent les réponses négatives de notre part à l'égard de cette chose. Cette escalade destructrice n'est point envisageable dans le cas de la pierre, car si cette dernière peut nous détruire voire nous tuer, ce n'est évidemment point parce qu'elle réagit à la négation

9. Bien que les animaux soient également doués de sentiment (selon E, III, 57, scolie), il ne sont pas semblables à l'homme et dès lors il ne saurait y avoir d'échange affectif avec eux (en vertu d'E, III, 33).

10. Puisque l'individualité est conférée par le corps (E, II, Lemme IV) et que les corps humains diffèrent sur beaucoup de points (E, I, Appendice). 
de sa propre essence mais simplement parce qu'elle entretient, au moment où elle nous détruit, un rapport qui ne s'accorde pas avec la nôtre. Ce rapport est invariable : il peut être reproduit autant de fois que les conditions qui le rendent possible sont réunies (il faut par exemple qu'une pierre d'un poids déterminé atteigne une vitesse déterminée pour blesser quelqu'un). Alors que la rencontre de l'homme avec les objets non rationnels met uniquement en jeu les compatibilités et incompatibilités de son essence avec ces derniers sous certains rapports invariables, les rencontres entre êtres humains soumis à l'imagination, au contraire, même si elles débutent elles aussi par des rapports de plus ou moins grande compatibilité, finissent nécessairement par la destruction, c'est-à-dire par le triomphe des passions tristes sur les passions joyeuses. Ceci veut dire que deux personnes soumises à l'imagination, risquent fortement de voir les affinités qui cimentaient au départ leur relation - par exemple une attirance mutuelle pour leur " forme belle » - être graduellement substituées par une haine réciproque ${ }^{11}$. Cette haine, nous l'avons vu, est engendrée par l'inévitable empiétement de " territoire » qu'entraîne le mécanisme de projection dans les relations intersubjectives gouvernées par l'imagination et des réactions que cet empiétement suscite mais surtout, elle est la conséquence de l'illusion de la liberté chez chacun des protagonistes impliqués dans ces relations. Car sans cette illusion, indissociable du mécanisme de projection, la haine entre ces protagonistes serait moins grande ${ }^{12}$ et par conséquent les risques d'une escalade destructrice le seraient également.

La réponse à la question de savoir pourquoi il faut quitter la dynamique projective de l'imagination est donc très claire : cette dernière rend extrêmement dangereuse la vie sociale des hommes et ceci d'autant plus que cette vie est pour eux une nécessité ${ }^{13}$. Ainsi le mal éthique par excellence dont il faut se guérir est l'imagination en tant qu'elle se déploie dans les relations intersubjectives, car c'est là que les dégâts qu'elle provoque sont les plus dommageables.

Nous espérons avoir pu montrer, dans le cadre de cet article, l'importance du mécanisme de projection imaginatif, au sein de la démarche éthique spinozienne. Nous pensons que l'analyse de ce dernier offre une voie d'accès privilégiée à cette démarche, parce qu'il concentre à lui seul l'enjeu fondamental de cette dernière, soit l'urgence éthique du salut face à la destruction inévitable de notre essence, lorsque nous vivons sous régime imaginatif. C'est cette urgence qui réclame de ne se concentrer que sur l'essentiel, soit précisément sur ce mécanisme projectif, dont le remède réside dans sa propre compréhension.

11. Sur ce point, cf. E, IV, Appendice, ch. 19.

12. En vertu d'E, III, 49.

13. Cf. E, IV, 35, scolie. 